\title{
Osteonecrosis of the Jaw: More Heat Than Light
}

O steonecrosis of the jaw has received a great deal of attention in the past few years. The earliest case reports claimed that the only time it ever occurred was as a complication of therapy with bisphosphonates and that both oral and intravenous bisphosphonates were implicated (1). These reports led to use of terms such as BRONJ (bisphosphonate-related osteonecrosis of the jaw). The speculation about pathogenic mechanisms, including any relationship with bisphosphonate therapy, has shifted in emphasis over time, starting with the notion that the mechanism might be a form of avascular necrosis of bone and more recently emphasizing the importance of

\section{See page 30}

infection, even leading to a suggestion (2) that the lesions be renamed osteomyelitis of the jaw, or bisphosphonateassociated osteomyelitis of the jaw.

Although osteonecrosis of the jaw is not a new clinical entity and has long been recognized in association with radiation or glucocorticoid use, no specific International Classification of Diseases code had been assigned to osteonecrosis of the jaw until recently. Problems with definition and lack of good epidemiologic data have led to considerable confusion, but progress is being made in placing the condition into perspective, and several recent reviews are available about osteonecrosis of the jaw and its

Received Sep. 9, 2008; revision accepted Sep. 16, 2008.

For correspondence or reprints contact: Ignac Fogelman, Guy's and St. Thomas' Hospital, St.

Thomas St., London, U.K. SE1 9RT.

E-mail: ignac.fogelman@kcl.ac.uk

COPYRIGHT (c) 2009 by the Society of Nuclear

Medicine, Inc.

DOI: 10.2967/jnumed.108.057885 pathogenesis (2-7) and about the actions of bisphosphonates (8). Although some clinical and pathologic features of osteonecrosis of the jaw continue to be debated, the current definition is of nonhealing (for $>6-8 \mathrm{wk}$ ) lesions involving exposed bone in the mandible or maxilla, usually after dental interventions such as tooth extraction.

It is now acknowledged that any risk that osteonecrosis of the jaw will occur in association with oral bisphosphonate use is extremely low (estimated to be $<1$ per 100,000 patients per year) and may be close to background rates of osteonecrosis of the jaw in untreated patients, even though these rates are not clearly defined $(3,9,10)$. This acknowledgment casts doubt on any causal relationship, which is reassuring in that there is no rationale for withholding treatment with bisphosphonates in osteoporosis when the clinical benefit of reducing fractures outweighs any perceived risk.

Most cases of osteonecrosis of the jaw have occurred in cancer patients, for whom incidences ranging from $1 \%$ to $5 \%$ or higher have been reported. Osteonecrosis of the jaw seems to occur especially in patients with myeloma or breast cancer metastases, and such patients are those who have to receive high-dose parenteral bisphosphonates (pamidronate or zoledronate) to prevent the skeletal complications of malignancy. The etiology of osteonecrosis is unknown, and recent reviews and guidelines emphasize that even in the cancer setting any causal role for bisphosphonates remains unproven, even though they are used at much higher doses in cancerous conditions than in benign conditions. Indeed, no prospective controlled trials have shown a significant association. Moreover no association has been found between bisphosphonate use and osteonecrosis at the more familiar sites of hip and knee, where several etiologic factors are recognized (e.g., steroids, fat embolism, and decompression sickness). Many cancer patients with osteonecrosis of the jaw receive other drugs, including glucocorticoids or chemotherapeutic agents, making the potential pathogenic mechanisms complex. Relevant prospective clinical trials or animal models to study pathogenic mechanisms are not available, and speculation continues about the roles of infection and immunodysregulation and about interference with angiogenesis and tissue healing $(5,11)$.

Regardless of the pathogenic mechanisms involved, the diagnosis of osteonecrosis of the jaw and its proper management remain clinical challenges, and progress in these directions is to be welcomed. In this volume of The Journal of Nuclear Medicine, Dore et al. (12) have attempted to throw some light on the complicated issue of establishing the diagnosis of osteonecrosis of the jaw, associated with the presence of necrotic lesions in the jaw. The investigators used panoramic dental radiographs, MRI or CT, and 3-phase bone scintigraphy with SPECT/CT in 15 patients, all of whom had cancer as well as clinically suspected osteonecrosis of the jaw. In addition, biopsies were performed on about half the individuals studied. As expected, the combination of these techniques was helpful in delineating areas of bone necrosis and regions of increased bone turnover. The findings are not at odds with any current thinking and suggest that in patients with increased ${ }^{99 \mathrm{~m}} \mathrm{Tc}-$ bisphosphonate uptake in the jaw, a bone SPECT/CT study may add some diagnostic value.

It is perhaps surprising that imaging techniques have not been more widely used for research into osteonecrosis of the jaw. A combination of 3-phase bone scintigraphy with SPECT/CT could be a useful investigative tool, but practical limitations, as Dore et al. outlined clearly, will restrict use as 
a routine test. Although it is encouraging to see that the panoramic dental radiographs, a routine imaging technique available in every dental practice, were able to pick up bone abnormalities in all patients studied, the findings were nonspecific.

Because functional change occurs before gross anatomic change, one would expect the isotope bone scan to have an advantage, but of course hot spots in the mandible and maxilla are frequently seen because of other common dental problems. However, the combination of SPECT/CT should provide optimal results. In this context, one should note that in the present study the SPECT/CT technology used was basic and is now a decade old, producing CT images that are $5-10 \mathrm{~mm}$ thick and of poor quality (but are useful in providing anatomic localization). More recent SPECT/CT systems provide greatly improved resolution with diagnostic capabilities. On the CT component of the study, a necrotic core was identified in 8 of the 15 subjects, and one would anticipate that the number would have been higher with a modern system. An important observation is that SPECT/CT provided valuable information in 7 patients who did not have exposed bone at the time they were studied (i.e., at that time they did not fulfill the criteria for osteonecrosis of the jaw) but the majority of whom had subsequently confirmed disease.

These imaging techniques assist in understanding some of the underlying processes. The uptake of ${ }^{99 \mathrm{~m}} \mathrm{Tc}$-labeled bisphosphonate is likely to reflect where bisphosphonates themselves localize. Moreover, tracer uptake will take place in areas of vascular access and in bone areas that are metabolically active, that is, regions where viable bone cells are laying down new miner- alizing bone matrix or undergoing resorption. Imaging with ${ }^{99 \mathrm{~m}} \mathrm{Tc}$ bisphosphonates does not support the assertion that the jaw bones are remodelling at rates that are substantially higher than other parts of the skeleton unless pathologic processes are taking place. Necrotic bone is nonviable and will not take up ${ }^{99 \mathrm{~m}} \mathrm{Tc}$-bisphosphonate. However, sites of surrounding metabolic activity, whether reflecting inflammatory changes or infection involving increased bone turnover, will take up isotope, as seen in this study. These techniques are therefore useful in distinguishing these processes and guiding appropriate treatment.

We conclude that SPECT/CT could indeed be helpful for managing osteonecrosis of the jaw (because of the ability to demonstrate the current functional status of the imaged area together with structural change) and for delineating viable from nonviable bone. SPECT/CT could thereby become the test of choice for diagnosing osteonecrosis of the jaw and for guiding surgical or other interventions. Nevertheless, in clinical practice all imaging techniques will inevitably be restricted to those patients in whom there is a high clinical suspicion of disease. It is to be hoped that continued research will further clarify the scale and clinical significance of these issues and encourage the appropriate management of patients (13).

Michael Pazianas
R. Graham G. Russell
Nuffield Orthopaedic Centre
Oxford, United Kingdom

Ignac Fogelman
Guy's Hospital
London, United Kingdom

\section{REFERENCES}

1. Marx RE. Oral and Intravenous BisphosphonateInduced Osteonecrosis of the Jaws: History, Etiology, Prevention, and Treatment. Hanover Park, IL: Quintessence Publishing Co., Inc.; 2007.

2. Wimalawansa SJ. Insight into bisphosphonateassociated osteomyelitis of the jaw: pathophysiology, mechanisms and clinical management. Expert Opin Drug Saf. 2008;7:491-512.

3. Khosla S, Burr D, Cauley J, et al. Bisphosphonateassociated osteonecrosis of the jaw: report of a task force of the American Society for Bone and Mineral Research. J Bone Miner Res. 2007;22: 1479-1491.

4. Pazianas M, Miller P, Blumentals WA, Bernal M, Kothawala P. A review of the literature on osteonecrosis of the jaw in patients with osteoporosis treated with oral bisphosphonates: prevalence, risk factors, and clinical characteristics. Clin Ther. 2007;29:1548-1558.

5. Reid IR, Bolland MJ, Grey AB. Is bisphosphonateassociated osteonecrosis of the jaw caused by soft tissue toxicity? Bone. 2007;41:318-320.

6. Rizzoli R, Burlet N, Cahall D, et al. Osteonecrosis of the jaw and bisphosphonate treatment for osteoporosis. Bone. 2008;42:841-847.

7. Van den Wyngaert T, Huizing MT, Vermorken JB. Bisphosphonates and osteonecrosis of the jaw: cause and effect or a post hoc fallacy? Ann Oncol. 2006;17:1197-1204.

8. Russell RG, Xia Z, Dunford JE, et al. Bisphosphonates: an update on mechanisms of action and how these relate to clinical efficacy. Ann N Y Acad Sci. 2007;1117:209-257.

9. Grbic JT, Landesberg R, Lin SQ, et al. Incidence of osteonecrosis of the jaw in women with postmenopausal osteoporosis in the health outcomes and reduced incidence with zoledronic acid once yearly pivotal fracture trial. $J$ Am Dent Assoc. 2008;139:32-40.

10. Bilezikian JP. Osteonecrosis of the jaw: do bisphosphonates pose a risk? N Engl J Med. 2006; 355:2278-2281.

11. Landesberg R, Cozin M, Cremers $S$, et al. Inhibition of oral mucosal cell wound healing by bisphosphonates. J Oral Maxillofac Surg. 2008;66: 839-847.

12. Dore F, Filippi L, Biasotto M, Chiandussi S, Cavalli F, Di Lenarda R. Bone scintigraphy and SPECT/CT of bisphosphonate-induced osteonecrosis of the jaw. J Nucl Med. 2009;50: 30-35.

13. Shane E, Goldring $\mathrm{S}$, Christakos $\mathrm{S}$, et al. Osteonecrosis of the jaw: more research needed. J Bone Miner Res. 2006;21:1503-1505. 